\title{
Pioglitazone Reduces ER Stress in the Liver: Direct Monitoring of in vivo ER Stress Using ER Stress-activated Indicator Transgenic Mice
}

\author{
KAZUTOMI YOSHIUCHI*, HIDEAKI KANETO*, TAKA-AKI MATSUOKA*, RYUICHI KASAMI*, \\ KENJI KOHNO**, TAKAO IWAWAKI***\#, YOSHIHISA NAKATANI*, YOSHIMITSU YAMASAKI*, \\ IICHIRO SHIMOMURA*AND MUNEHIDE MATSUHISA*
}

*Department of Metabolic Medicine, Osaka University Graduate School of Medicine, 2-2 Yamadaoka, Suita, Osaka 565-0871, Japan

** Graduate School of Biological Sciences, Nara Institute of Science and Technology, 8916-5 Takayama, Ikoma, Nara 630-0192, Japan

*** Iwawaki Initiative Research Unit, Frontier Research System, RIKEN, 2-1 Hirosawa, Wako, Saitama 351-0198, Japan

${ }^{\#}$ PRESTO, Japan Science and Technology Agency, 4-1-8 Honcho Kawaguchi, Saitama 332-0012, Japan

\begin{abstract}
It is known that endoplasmic reticulum (ER) stress is provoked under diabetic conditions and is possibly involved in the development of insulin resistance. In this study, using ER stress-activated indicator (ERAI) transgenic mice which express green fluorescent protein under ER stress conditions, we directly evaluated the effects of a diabetic agent pioglitazone on in vivo ER stress under diabetic conditions. In high fat and high sucrose diet-induced diabetic ERAI transgenic mice, 8 weeks of pioglitazone treatment reduced the accumulation of fat droplets in the liver and attenuated the development of insulin resistance. In the liver of the ERAI transgenic mice, ERAI fluorescence activity was clearly reduced as early as after 4 weeks of pioglitazone treatment, preceding the improvement of insulin resistance. In addition, after the pioglitazone treatment, serum free fatty acid and triglyceride levels were decreased, and serum adiponectin levels were increased. These data indicate that pioglitazone treatment suppresses ER stress in the liver which may explain, at least in part, the pharmacological effects of pioglitazone to reduce insulin resistance.
\end{abstract}

Key words: Type 2 diabetes, Insulin resistance, ER stress, Pioglitazone

(Endocrine Journal 56: 1103-1111, 2009)

THE ENDOPLASMIC RETICULUM (ER) is an organelle that synthesizes various secretory and membrane proteins. Under normal conditions, these proteins are correctly folded and assembled by chaperones in the ER, but under stressful conditions, the chaperones become overloaded and the ER fails to fold newly synthesized proteins, leading to the induction of ER stress [1-5]. ER stress can be provoked by accumulation of unfolded and misfolded proteins that aggregate in the ER lumen which occurs under a variety of pathophysiological conditions [6-9]. To maintain ER

Received May 11, 2009; Accepted Sep. 17, 2009 as K09E-140 Released online in J-STAGE as advance publication Sep. 29, 2009 Correspondence to: Hideaki KANETO, M.D., Ph.D., Department of Metabolic Medicine, Osaka University Graduate School of Medicine, 2-2 Yamadaoka, Suita, Osaka 565-0871, Japan.

E-mail: kaneto@medone.med.osaka-u.ac.jp function, cells activate a self-protective mechanism to survive ER stress conditions, which is known as unfolded protein response (UPR). This response consists of three different mechanisms: translational attenuation to limit further accumulation of unfolded proteins, transcriptional activation of genes encoding molecular chaperones, and ER-associated degradation (ERAD). When these adaptive responses are not sufficient to relive cells from ER stress, cells undergo apoptosis to destroy the ER stress-damaged cells themselves.

Type 2 diabetes mellitus is a complex disease characterized by insulin resistance and insufficient insulin secretion [10, 11]. Recently, various studies have shown that ER stress plays a crucial role in the development of insulin resistance and the pathogenesis of type 2 diabetes [12-19]. X-box-binding protein-1 (XBP-1) is a key transcription factor in the ER stress 
response through its transcriptional regulation of various genes, including molecular chaperones, and it was reported that mice deficient in XBP-1 develop insulin resistance [16]. Also, we recently found that ER stress is provoked in the liver at relatively early stage during the development of insulin resistance [20]. In addition, it was reported that oxygen-regulated protein 150 (ORP150), a molecular chaperone in the ER, protects cells from ER stress and that ORP150 overexpression improves insulin resistance and ameliorates glucose intolerance in diabetic animals $[17,18]$. These results suggest that ER stress is involved in the development of insulin resistance found in type 2 diabetes. In addition, it has recently been reported that induction of ER stress leads to the activation of the c-Jun N-terminal kinase (JNK) pathway which is also closely associated with the development of insulin resistance [21-25].

Pioglitazone is a ligand for the peroxisome proliferator-activated receptor $\gamma(\operatorname{PPAR} \gamma)$ which is a member of the nuclear receptor superfamily of ligand-regulated transcription factors and is implicated in a variety of physiological and pathological process including insulin resistance and type 2 diabetes [26-32]. Also, pioglitazone has been widely used as an insulin sensitizer in a clinical medicine. In the present study, to examine whether pioglitazone suppresses ER stress in the liver, we directly monitored in vivo ER stress using the ER stress-activated indicator (ERAI) transgenic mice.

\section{Subjects and Methods}

\section{ER stress-activated indicator (ERAI) transgenic mice}

To examine whether pioglitazone suppresses ER stress in the liver, we used ERAI transgenic mice carrying the XBP-1-delta-DBD-venus expression gene [33]. It is noted here that XBP-1 is a key transcription factor in the ER stress response through its transcriptional regulation of various genes, including molecular chaperones. In these transgenic mice, the gene encoding venus, a variant of green fluorescent protein, is fused as a reporter downstream of a partial sequence of human XBP-1 including the ER stress-specific intron. Under normal conditions, mRNA of the fusion gene is not spliced and its translation is terminated at the stop codon near the junction between the $X B P-1$ and venus genes. In contrast, under ER stress conditions, the intron is spliced out, which leads to a frameshift of the fusion mRNA, and translation of the spliced mRNA continues up to the stop codon of venus. Thereby, under diabetic conditions, a fusion protein of XBP-1 and venus is produced in cells and can be detected by monitoring the fluorescence activity of venus. In this study, the ERAI transgenic mice were randomized into two groups at 4 weeks of age: high fat and high sucrose (HF/HS) diet with and without $0.02 \%$ pioglitazone groups (approximately $20 \mathrm{mg} /$ $\mathrm{kg} /$ day), and kept under controlled lighting conditions (12 h light and $12 \mathrm{~h}$ dark). Pioglitazone was kindly provided by Takeda Chemical Institutes Co., Ltd., (Osaka, Japan). The experimental protocol was approved by the Ethics Review Committee for Animal Experimentation of Osaka University Graduate School of Medicine.

\section{Glucose tolerance tests}

After an overnight fast, mice were injected intraperitoneally with glucose (2.0 $\mathrm{g} / \mathrm{kg}$ body weight). Blood samples were taken at various time points (0-120 min), and blood glucose levels were measured regularly with a portable glucose meter (Glu-test Sensor; Sanwa, Osaka, Japan) after tail snipping.

\section{Measurement of serum insulin levels}

Blood samples were collected into heparinized capillary tubes, and serum insulin levels were determined with the Insulin-ELISA Kit/Mouse Ultra Sensitive (Morinaga Biochemicals).

\section{Insulin tolerance tests}

After a 3-h fast, mice were injected intraperitoneally with insulin (1.0 units $/ \mathrm{kg})$. Blood samples were taken at various time points (0-90 min), and blood glucose levels were measured regularly with a portable glucose meter after tail snipping.

\section{Observation of tissues}

ERAI transgenic mice were injected with sodium pentobarbital (Nembutal: $10 \mathrm{mg} / \mathrm{kg}$, i.p.), and various tissues were removed from the mice. Tissue samples were immersed in $4 \%$ (wt/vol) buffered paraformaldehyde (PFA) for $16 \mathrm{~h}$ and then in $10 \%, 20 \%$, and $30 \%$ sucrose for $8 \mathrm{~h}$ each at $4{ }^{\circ} \mathrm{C}$. To confirm ERAI fluo- 
rescence activity in tissue samples, representative tissue sample segments were embedded in frozen section media (OCT medium, Tissue-Tek), sectioned $(5 \mu \mathrm{m})$ and observed with a fluorescent microscope model Nikon Eclipse 80 i; then pictures were taken with the Nikon ACT-1 ver. 2.63.

\section{Western blot analysis}

Whole cell extracts obtained from the liver were fractionated by $10 \%$ SDS-PAGE and transferred to nitrocellulose membrane (Bio-Rad Laboratories, Inc.). After blocking, the membranes were incubated at 4 ${ }^{\circ} \mathrm{C}$ overnight in TBS buffer (50 mM Tris-HCl, 150 $\mathrm{mM} \mathrm{NaCl}$ ) containing a 1:1000 dilution of rabbit antiBip (GRP78) antibody (Santa Cruz Biotechnology), 1:1000 dilution of mouse anti-KDEL antibody (Stress Gene), or 1:1000 dilution of rabbit anti-PEPCK antibody (Santa Cruz Biotechnology), and then incubated for $1 \mathrm{~h}$ at room temperature in TBS containing a HRPconjugated secondary antibody (Bio-Rad Laboratories, Inc.). Immunoreactive bands were visualized by incubation with LumiGLO (Cell Signaling) and exposed to light-sensitive film.

Measurement of serum free fatty acid (FFA), triglyceride (TG), and adiponectin levels

Serum FFA and TG levels were assayed by enzymatic methods (SRL Co., Ltd., Tokyo, Japan), and serum adiponectin levels were measured with a mouse adiponectin enzyme-linked immunosorbent assay kit (Otsuka Pharmaceutical Co., Ltd., Tokyo, Japan).

\section{Measurement of TG content in the liver}

After addition of $2 \mathrm{M} \mathrm{NaCl} / 2 \mathrm{mM}$ EDTA / $50 \mathrm{mM}$ sodium phosphate buffer, $\mathrm{pH}$ 7.4, liver samples were sonicated for 1-2 min and centrifuged at 12,000 rpm for $5 \mathrm{~min}$. The amounts of liver TG were measured using a 'TG-EN Kainos' kit (Kainos, Tokyo, Japan).

\section{Statistical analysis}

Results are expressed as means \pm SE. Differences between groups were examined for statistical significance using the Student's $t$ test or analysis of variance with the Fisher's test. ${ }^{*} p<0.05$

\section{Results and Discussion}

Pioglitazone treatment reduces high fat and high sucrose $(\mathrm{HF} / \mathrm{HS})$ diet-induced insulin resistance in $E R$ stress-activated indicator (ERAI) transgenic mice.

To directly evaluate the effects of pioglitazone on ER stress in the liver under diabetic conditions with insulin resistance, we treated ERAI transgenic mice with a HF/HS diet which is well known to induce insulin resistance. Since ERAI transgenic mice express green fluorescent protein (GFP) under ER stress conditions, we evaluated ER stress levels in vivo after pioglitazone treatment in HF/HS diet-induced diabetic ERAI transgenic mice.

As shown in Fig.1A, there was no difference in body weight of HF/HS diet-induced diabetic ERAI transgenic mice between with and without pioglitazone treatment. First, to examine the effects of pioglitazone treatment on glucose tolerance in $\mathrm{HF} / \mathrm{HS}$ diet-induced diabetic ERAI transgenic mice, we performed an intraperitoneal glucose tolerance test (IPGTT). As shown in Fig. 1B, after 4 weeks of pioglitazone treatment, glucose tolerance was almost same as that without pioglitazone treatment. After 8 weeks of pioglitazone treatment, however, glucose tolerance was moderately ameliorated compared to that without pioglitazone treatment. In addition, as shown in Fig. 1C, after pioglitazone treatment, fasting serum insulin levels were lower compared to those without pioglitazone treatment, although it did not reach a statistical significance.

Next, to evaluate the effects of pioglitazone on insulin resistance in HF/HS diet-induced diabetic ERAI transgenic mice, we performed an intraperitoneal insulin tolerance test (IPITT). As shown in Fig. 1D, after 4 weeks of pioglitazone treatment, blood glucose levels after insulin load were almost same as those without pioglitazone treatment. After 8 weeks of pioglitazone treatment, however, the reduction of blood glucose levels after insulin load was significantly larger compared to that without pioglitazone. These results indicate that 8 weeks of pioglitazone treatment actually reduced insulin resistance in HF/HS diet-induced diabetic ERAI transgenic mice. In addition, to examine the effects of pioglitazone treatment on gluconeogenesis, we evaluated the expression level of key gluconeogenic enzyme phosphoenolpyruvate carboxykinase (PEPCK) in the liver by western blot analysis but failed to detect the difference in PEPCK 
A
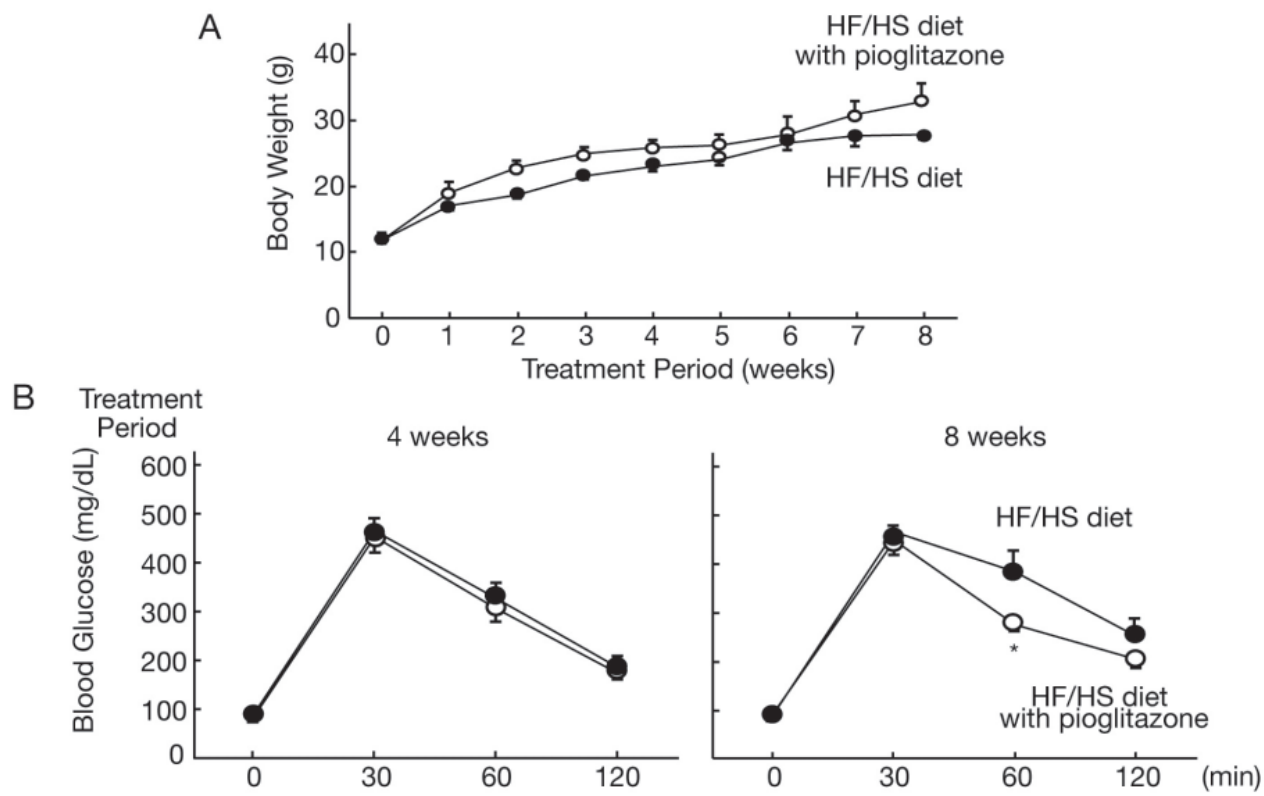

C Treatment
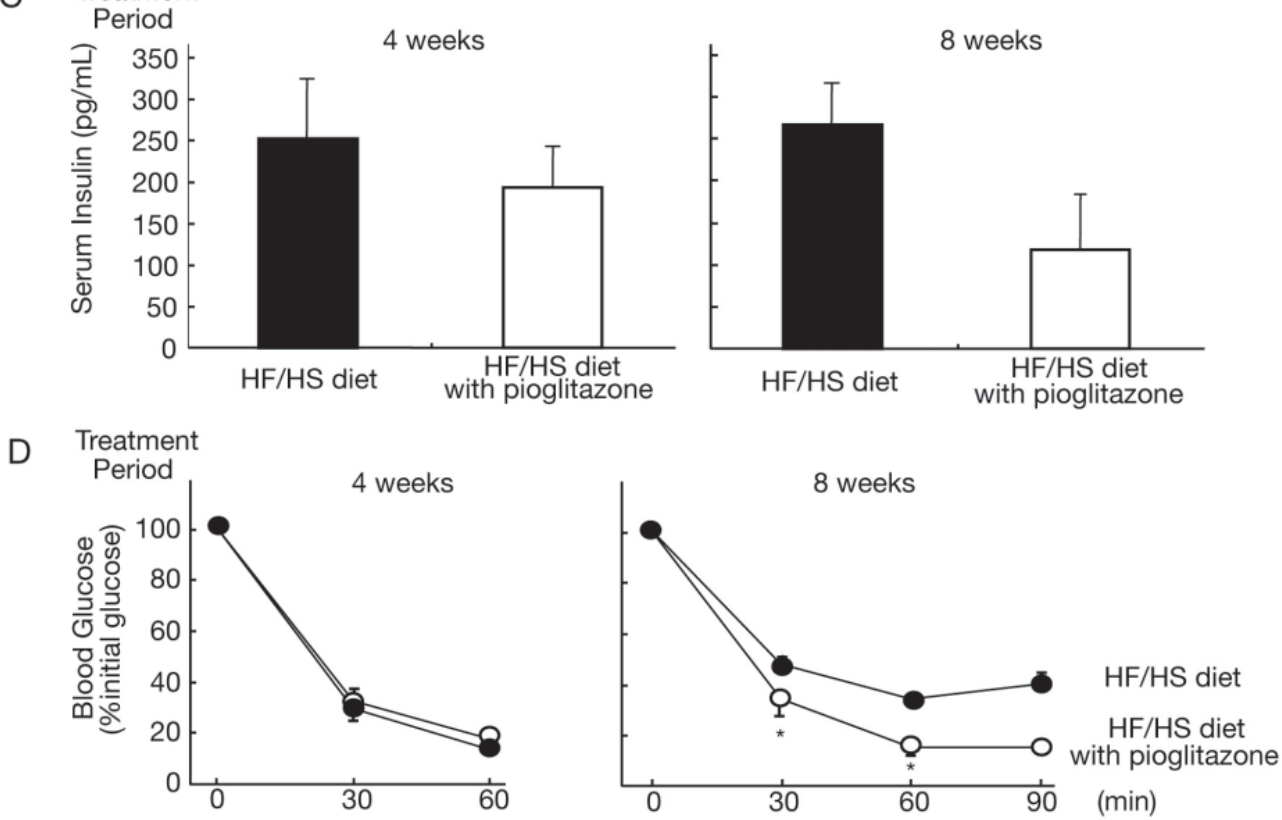

Fig. 1. Effects of pioglitazone treatment on HF/HS diet-induced insulin resistance in ERAI transgenic mice.

(A) There was no difference in body weight of HF/HS diet-induced diabetic ERAI transgenic mice between with and without pioglitazone treatment. Closed circle, HF/HS diet group; open circle, HF/HS diet with pioglitazone group. Data are shown as means \pm S.E. $(n=5-19)$. (B) An intraperitoneal glucose tolerance test was performed after pioglitazone treatment in HF/HS dietinduced diabetic mice. After 4 weeks of pioglitazone treatment, glucose tolerance was almost same as that without pioglitazone treatment. After 8 weeks of pioglitazone treatment, however, glucose tolerance was ameliorated compared to that without pioglitazone treatment. Closed circle, HF/HS diet group; open circle, HF/HS diet with pioglitazone group. Data are shown as means \pm S.E. $(n=4-10) * p<0.05$. (C) After pioglitazone treatment, fasting serum insulin levels were decreased, although it did not reach a statistical significance. Data are shown as means \pm S.E. $(n=4-10)$ (D) An intraperitoneal insulin tolerance test was performed after pioglitazone treatment in HF/HS diet-induced diabetic mice. After 4 weeks of pioglitazone treatment, blood glucose levels after insulin load were almost same as those without pioglitazone treatment. After 8 weeks of pioglitazone treatment, however, the reduction of blood glucose levels was significantly larger compared to that without pioglitazone treatment. Closed circle, HF/HS diet group; open circle, HF/HS diet with pioglitazone group. Data are shown as means \pm S.E. $(n=6-8) * p<0.05$. 

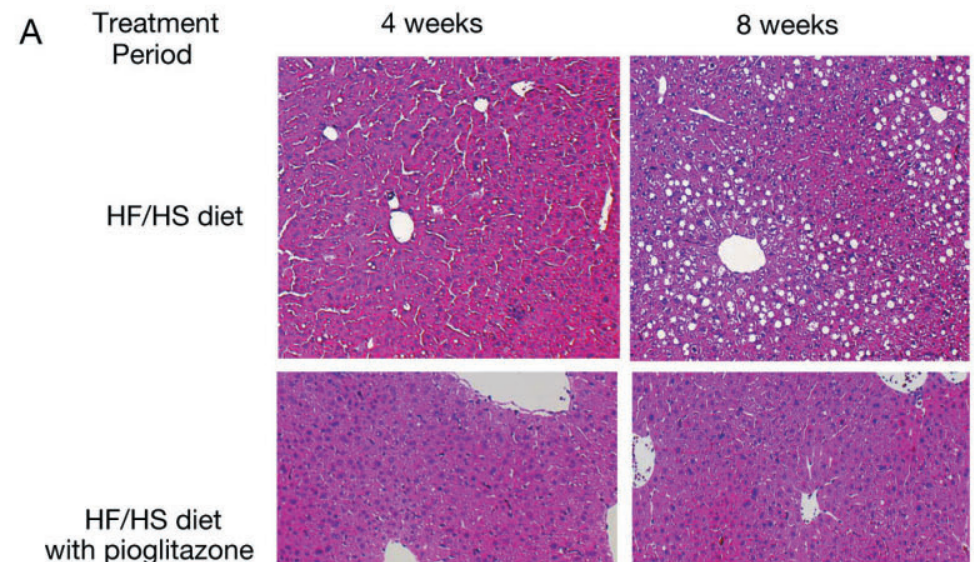
with pioglitazone
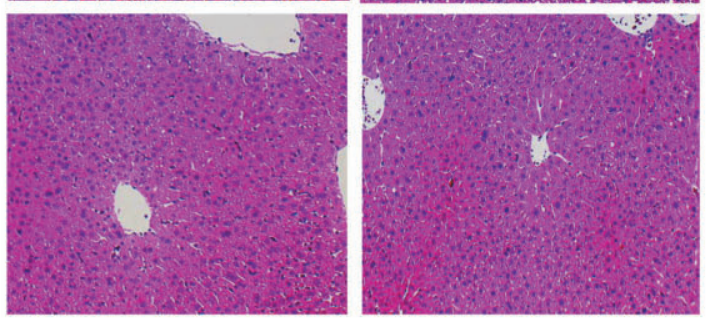

\section{B

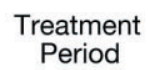

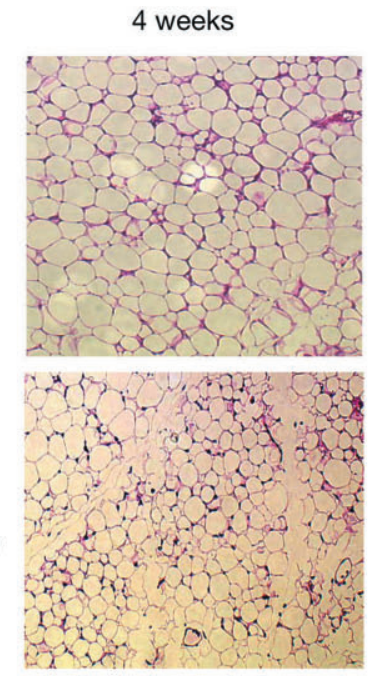

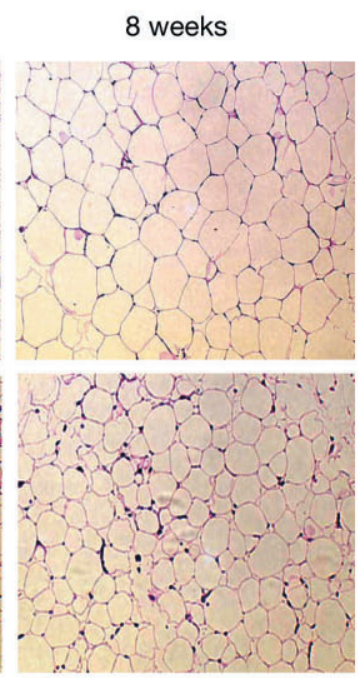

Fig. 2. Effects of pioglitazone treatment on HF/HS diet-induced morphological changes in the liver and fat of ERAI transgenic mice.

(A) Hematoxylin and eosin (HE) staining was performed after pioglitazone treatment in HF/HS diet-induced diabetic ERAI transgenic mice. After 4 weeks of HF/HS diet treatment, there is no clear morphological difference in the liver of the transgenic mice between with and without pioglitazone treatment. After 8 weeks of HF/HS diet treatment, the liver is vacuolized due to accumulation of fat droplets, whereas such a change is clearly reduced by pioglitazone treatment. (B) Smaller adipocytes are observed after pioglitazone treatment compared to those without pioglitazone treatment in HF/HS diet-induced diabetic ERAI transgenic mice.

expression levels between the two groups (data not shown).

Pioglitazone treatment reduces the accumulation of fat droplets in the liver and the size of adipocytes in HF/ HS diet-induced diabetic ERAI transgenic mice.

To investigate the morphological change in the liver by pioglitazone, we performed hematoxylin and eosin (HE) staining after pioglitazone treatment. As shown in Fig. 2A, after 4 weeks of HF/HS diet treatment, there was no clear morphological difference in the liver of the transgenic mice between with and without pioglitazone treatment. After 8 weeks of $\mathrm{HF} / \mathrm{HS}$ diet treatment, the liver was vacuolized due to accumulation of fat droplets, whereas such a change was markedly reduced by pioglitazone treatment. Moreover, triglyceride content in the liver after 8 weeks of pioglitazone treatment was lower compared to that without pioglitazone treatment (32.6 \pm 2.6 v.s. $50.9 \pm 9.7 \mathrm{mg} / \mathrm{g}$ tissue). 


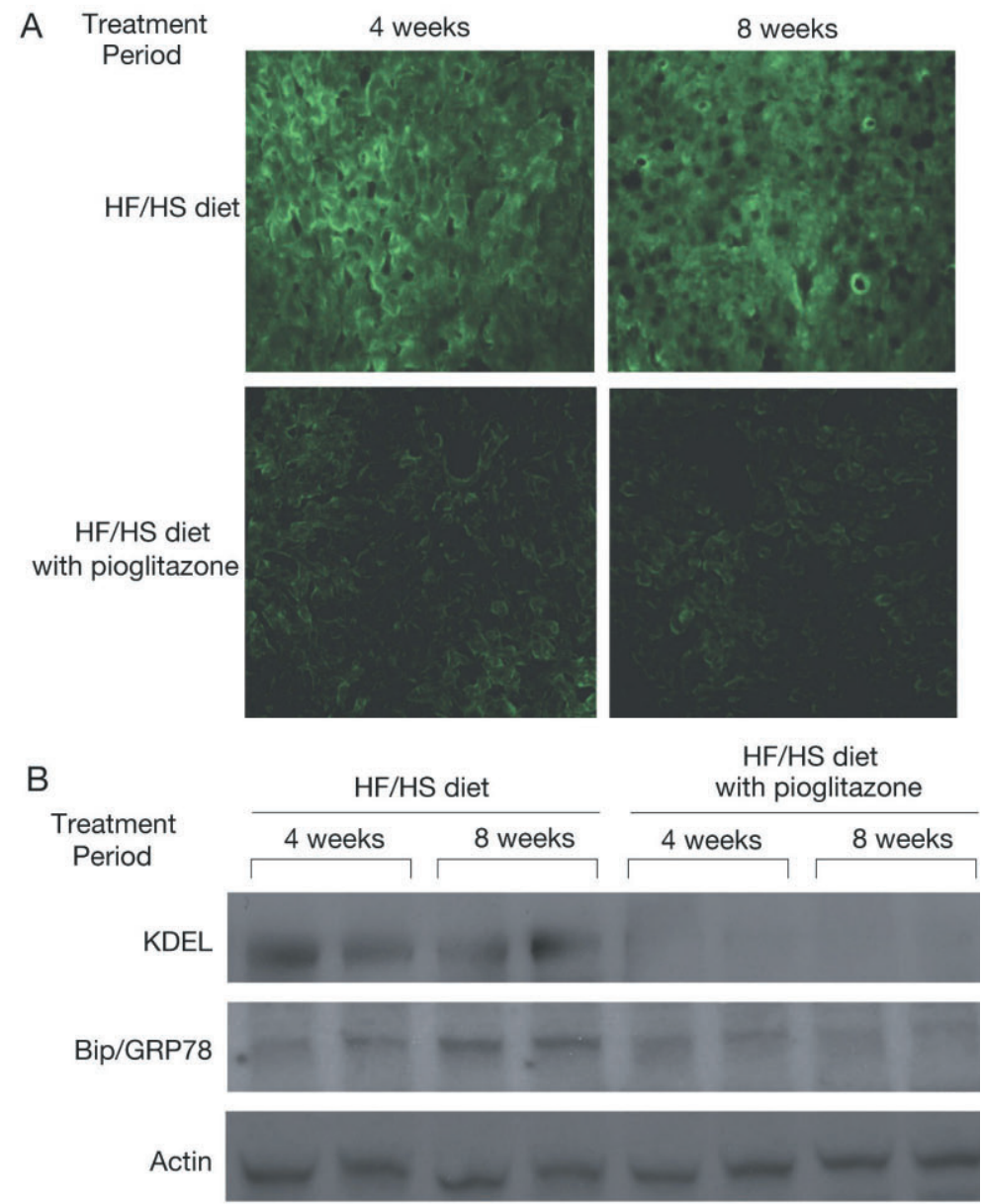

Fig. 3. Effects of pioglitazone treatment on HF/HS diet-induced ER stress in the liver of ERAI transgenic mice.

(A) ERAI fluorescence activity is clearly observed in the liver of ERAI transgenic mice after 4 weeks of HF/HS diet treatment, and stronger ERAI fluorescence activity is observed after 8 weeks of HF/HS diet treatment. In contrast, ERAI fluorescence activity is markedly reduced in the liver as early as after 4 weeks of pioglitazone treatment. Even after 8 weeks of HF/HS diet treatment, ERAI fluorescence activity is markedly reduced in the liver by pioglitazone treatment.

(B) KEDL and Bip/GPR78 expression, both of which are well known as ER stress markers, were clearly detected in the liver after 4 and 8 weeks of HF/HS diet treatment. In contrast, after 4 and 8 weeks of pioglitazone treatment, levels of KEDL and Bip/GPR78 expression were reduced compared to those without pioglitazone treatment.

Next, to investigate the morphological change in the fat by pioglitazone, we performed HE staining after pioglitazone treatment. As shown in Fig. 2B, in HE staining, smaller adipocytes were observed after pioglitazone treatment compared to those without pioglitazone treatment.

Pioglitazone treatment suppresses HF/HS diet inducedER stress in the liver of ERAI transgenic mice.

To examine whether pioglitazone suppresses ER stress in the liver, we directly monitored in vivo ER stress using the HF/HS diet-induced diabetic ERAI transgenic mice. As shown in Fig. 3A, ERAI fluorescence activity was clearly observed in the liver after 4 weeks of HF/HS diet treatment, and stronger ERAI fluorescence activity was observed after 8 weeks of HF/ HS diet treatment. In contrast, ERAI fluorescence activity was markedly reduced in the liver as early as after 4 weeks of pioglitazone treatment. Even after 8 weeks of HF/HS diet treatment, ERAI fluorescence activity was markedly reduced in the liver by pioglitazone treatment. There was no difference in ERAI fluorescence activity in the liver between 4 and 8 weeks of pioglitazone treatment. Next, to confirm whether this ERAI fluorescence activity of this model correctly 
A Treatment
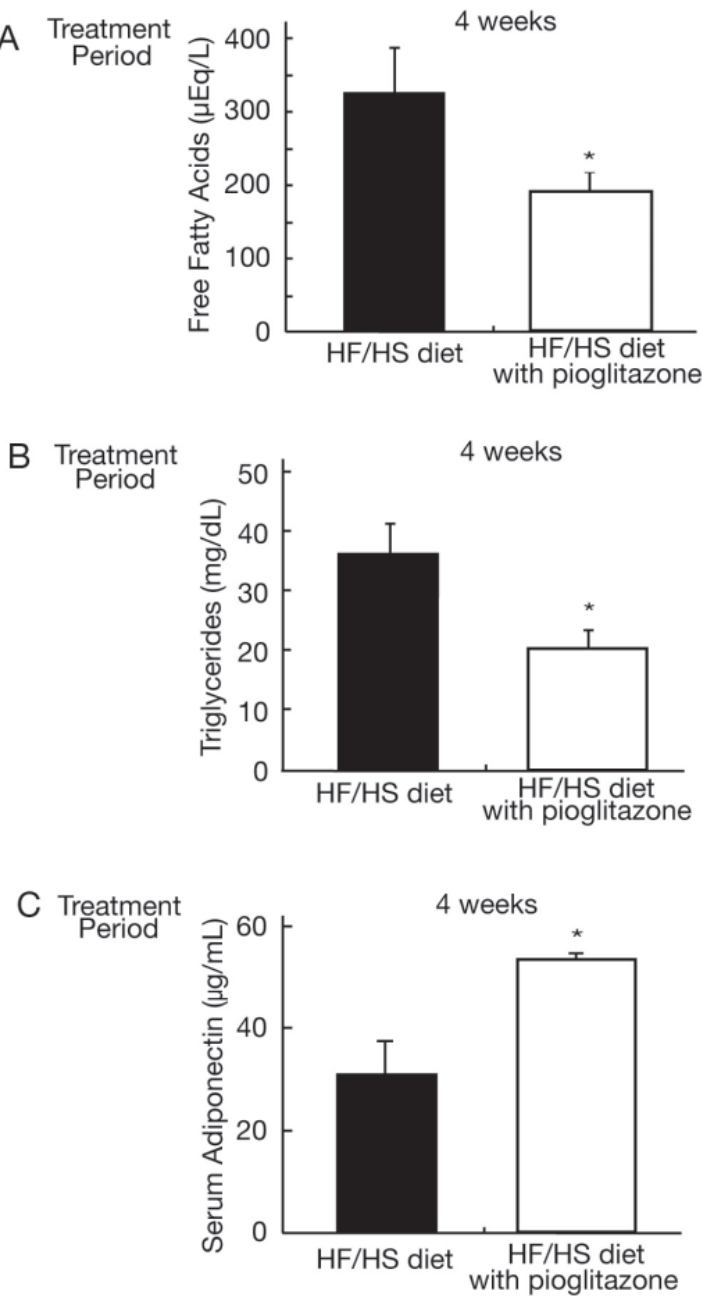
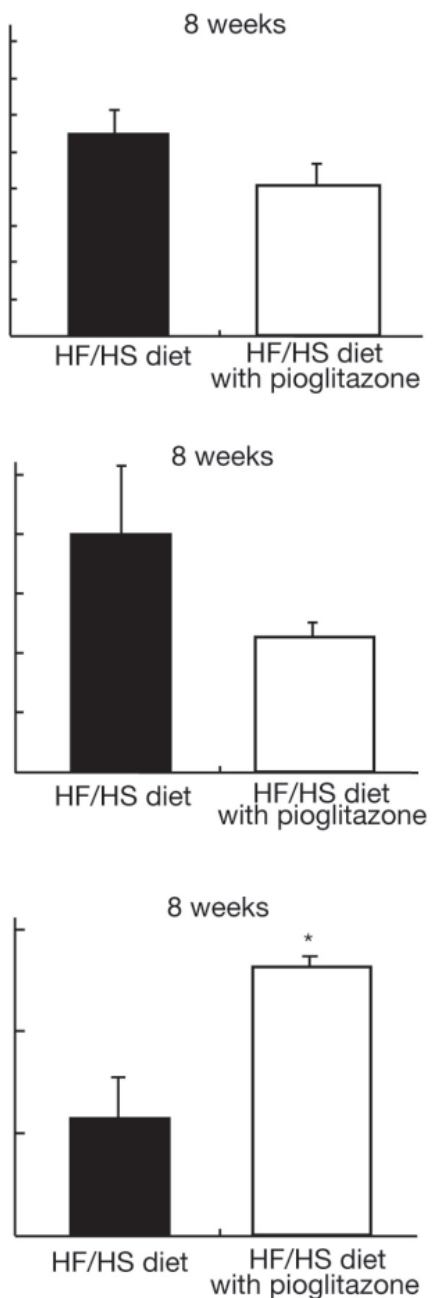

Fig. 4. Effects of pioglitazone treatment on the lipid metabolism in HF/HS diet-induced diabetic ERAI transgenic mice.

(A, B) After pioglitazone treatment, serum levels of FFA and triglycerides were decreased compared to those without pioglitazone treatment. Closed square, HF/HS diet group; open square, HF/HS diet with pioglitazone group. Data are shown as means \pm S.E. $(n=3-15) * p<0.05$ (C) After pioglitazone treatment, serum adiponectin levels were significantly higher compared to those without pioglitazone treatment. Closed square, HF/HS diet group; open square, HF/HS diet with pioglitazone group. Data are shown as means \pm S.E. $(n=3-7) * p<0.05$.

reflects the ER stress status, we examined expression levels of several ER stress markers by western blot analysis. As shown in Fig. 3B, KEDL and Bip/GPR78 expression, both of which are well known as ER stress markers, were clearly detected in the liver after 4 and 8 weeks of HF/HS diet treatment. In contrast, after 4 and 8 weeks of pioglitazone treatment, levels of KEDL and Bip/GPR78 expression were reduced compared to those without pioglitazone treatment. These results suggest that the ERAI fluorescence activity of this model is correlated with the ER stress status. On the other hands, ERAI fluorescence activity was not observed in the fat after the HF/HS diet treatment. These results suggest that pioglitazone treatment improves insulin resistance, at least in part, by reducing ER stress.

Pioglitazone treatment decreases serum FFA and triglyceride levels and increases serum adiponectin levels in HF/HS diet-treated ERAI transgenic mice.

To examine the effects of pioglitazone on lipid metabolism, we measured serum lipid profiles such as serum FFA and triglyceride levels and serum adiponectin levels in HF/HS diet-induced diabetic ERAI transgenic mice with and without pioglitazone treatment. As shown in Fig. 4A and 4B, serum levels of 
FFA and triglyceride after pioglitazone treatment were lower compared to those without pioglitazone treatment. In contrast, serum adiponectin levels after pioglitazone treatment were higher compared to those without the treatment (Fig. 4C). Therefore, we think it is possible that decreased levels of serum FFAs and triglycerides and increased levels of serum adiponection influenced the reduction of ER stress in the liver.
Taken together, pioglitazone treatment suppresses ER stress in the liver and the reduction of ER stress may explain, at least in part, the pharmacological effects of pioglitazone to reduce insulin resistance and that the ERAI transgenic mice are useful for evaluating the effects of various reagents on in vivo ER stress, especially in the liver.

\section{References}

1. Lee AS. (1992) Mammalian stress response: induction of the glucose-regulated protein family. Curr Opin Cell Biol 4: 267-273.

2. Gething MJ, Sambrook J. (1992) Protein folding in the cell. Nature 355: 33-45.

3. Kaufman RJ. (1999) Stress signaling from the lumen of the endoplasmic reticulum: coordination of gene transcriptional and translational condition. Genes Dev 13: 1211-1233.

4. Aridor M, Balch WE. (1999) Integration of endoplasmic reticulum signaling in health and disease. $N a t$ Med 5: 745-751.

5. Ron D. (2002) Translational control in the endoplasmic reticulum stress response. J Clin Invest 110: 13831388.

6. Hotamisligil GS. (2005) Role of endoplasmic reticulum stress and c-Jun NH2-terminal kinase pathways in inflammation and origin of obesity and diabetes. Diabetes 54: S73-78.

7. Kaneto H, Matsuoka T, Nakatani Y, Kawamori D, Miyatsuka T, Matsuhisa M, Yamasaki Y. (2005) Oxidative stress, ER stress, and the JNK pathway in type 2 diabetes. J Mol Med 83: 429-439.

8. Hotamisligil GS. (2006) Inflammation and metabolic disorders. Nature 444: 860-867.

9. Marciniak SJ, Ron D. (2006) Endoplasmic reticulum stress signaling in disease. Physiol Rev 86: 1133-1149.

10. Saltiel AR, Kahn CR. (2001) Insulin signalling and the regulation of glucose and lipid metabolism. Nature 414: 799-806.

11. Shulman GI. (2006) Cellular mechanisms of insulin resistance. J Clin Invest 106: 171-176.

12. Delepine M, Nicolino M, Barrett T, Golamaully M, Lathrop MG, Julier C. (2000) EIF2AK3, encoding translation initiation factor 2-alpha kinase 3, is mutated in patients with Wolcott-Rallison syndrome. Nat Genet 25: 406-409.

13. Harding HP, Zhang Y, Jungries R, Chung P, Plesken H, Sabatini DD, Ron D. (2001) Diabetes mellitus and exocrine pancreatic dysfunction in perk-/- mice reveals a role for translational control in secretory cell survival.
Mol Cell 7: 1153-1163.

14. Allen JR, Nguyen LX, Sargent KE, Lipson KL, Hackett A, Urano F. (2004) High ER stress in beta-cells stimulates intracellular degradation of misfolded insulin. Biochem Biophys Res Commun 324: 166-170.

15. Nozaki J, Kubota H, Yoshida H, Naitoh M, Goji J, Yoshinaga T, Mori K, Koizumi A, Nagata K. (2004) The endoplasmic reticulum stress response is stimulated through the continuous activation of transcription factors ATF6 and XBP1 in Ins2 ${ }^{+/ \text {Akita }}$ pancreatic $\beta$ cells. Genes Cells 9: 261-270.

16. Ozcan U, Cao Q, Yilmaz E, Lee AH, Iwakoshi NN, Ozdelen E, Tuncman G, Gorgun C, Glimcher LH, Hotamisligil GS. (2004) Endoplasmic reticulum stress links obesity, insulin action, and type 2 diabetes. Science 306: 457-461.

17. Nakatani Y, Kaneto H, Kawamori D, Yoshiuchi K, Hatazaki M, Matsuoka T, Ozawa K, Ogawa S, Hori M, Yamasaki Y, Matsuhisa M. (2005) Involvement of endoplasmic reticulum stress in insulin resistance and diabetes. J Biol Chem 280: 847-851.

18. Ozawa K, Miyazaki M, Matsuhisa M, Takano K, Nakatani Y, Hatazaki M, Tamatani T, Yamagata K, Miyagawa J, Kitao Y, Hori O, Yamasaki Y, Ogawa S. (2005) The endoplasmic reticulum chaperone improves insulin resistance in type 2 diabetes. Diabetes 54: 657663.

19. Ozcan U, Yilmaz E, Ozcan L, Furuhashi M, Vailancourt E, Smith RO, Gorgun C, Hotamisligil GS. (2006) Chemical chaperones reduce ER stress and restore glucose homeostasis in a mouse model of type 2 diabetes. Science 313: 1137-1140.

20. Yoshiuchi K, Kaneto H, Matsuoka T, Kohno K, Iwawaki T, Nakatani Y, Yamasaki Y, Hori M, Matsuhisa M. (2008) Direct monitoring of in vivo ER stress during the development of insulin resistance with ER stress-activated indicator transgenic mice. Biochem Biophys Res Commun 366: 545-550.

21. Urano F, Wang X, Bertolotti A, Zhang Y, Chung P, Harding HP. (2000) Coupling of stress in the ER to activation of JNK protein kinases by transmembrane pro- 
tein kinase IRE1. Science 287: 664-666.

22. Aguirre V, Davis R, White MF. (2000) The c-Jun $\mathrm{NH}_{2}-$ terminal kinase promotes insulin resistance during association with insulin receptor substrate-1 and phosphorylation of $\mathrm{Ser}^{307}$. J Biol Chem 275: 9047-9054.

23. Hirosumi J, Tuncman G, Chang L, Karin M, Hotamisligil GS. (2002) A central role for JNK in obesity and insulin resistance. Nature 420: 333-336.

24. Kaneto H, Nakatani Y, Miyatsuka T, Kawamori D, Matsuoka T, Matsuhisa M, Kajimoto Y, Ichijo H, Yamasaki Y, Hori M. (2004) Possible novel therapy for diabetes with cell-permeable JNK inhibitory peptide. Nat Med 10: 1128-1132.

25. Nakatani Y, Kaneto H, Kawamori D, Hatazaki M, Miyatsuka T, Matsuoka T, Kajimoto Y, Matsuhisa M, Yamasaki Y, Hori M. (2004) Modulation of the JNK pathway in liver affects insulin resistance status. $J$ Biol Chem 279: 45803-45809.

26. Mangelsdorf DJ, Thummel C, Beato M, Chambon P, Evans RM. (1995) The nuclear receptor superfamily: the second decade. Cell 83: 835-839.

27. Rosen ED, Spiegelman BM. (2001) PPAR- $\gamma$ : A nuclear receptor of metabolism, differentiation and cell growth. J Biol Chem 276: 37731-37734.

28. Moller DE, Berger JP. (2003) Role of PPARs in the regulation of obesity-related insulin sensitivity and inflammation. Int J Obes 7: S17-S21.

29. Kubota N, Terauchi Y, Kubota T, Kumagai H, Itoh S, Satoh H. (2006) Pioglitazone Ameliorates Insulin Resistance and Diabetes by Both Adiponectindependent and -independent Pathways. J Biol Chem 281: 8748-8755.

30. Rasouli N, Tao-Borengasser A, Miles LM, Elbein SC, Kern PA. (2006) Increased plasma adiponectin in response to pioglitazone does not result from increased gene expression. Am J Physiol Endocrinol Metab 290: E42-E46.

31. Loffler M, Bilban M, Reimers M, Waldhausl W, Stulnig TM. (2006) Blood glucose-lowering nuclear receptor agonists only partially normalize hepatic gene expression in $d b / d b$ mice, $J$ Pharmacol Exp Ther 316: 797-804.

32. Han KL, Choi JS, Lee JY, Song J, Joe MK, Jung MH, Hwang JK. (2008) Therapeutic potential of peroxisome proliferators-activated receptor-alpha/ gamma dual agonist with alleviation of endoplasmic reticulum stress for the treatment of diabetes. Diabetes 57: 737-745.

33. Iwawaki T, Akai R, Kohno K, Miura M. (2004) A transgenic mouse model for monitoring endoplasmic reticulum stress. Nat Med 10: 98-102. 